\title{
Eigenvibrations of a beam with two mechanical resonators attached to the ends
}

\author{
L. N. Koronova ${ }^{1}, D . M$. Korosteleva ${ }^{2}, K . O$. Levinskaya $^{1}$, and S. I. Solov'ev ${ }^{1, *}$ \\ ${ }^{1}$ Kazan Federal University, 18 Kremlevskaya Street, Kazan, 420008, Russian Federation \\ ${ }^{2}$ Kazan State Power Engineering University, 51 Krasnoselskaya Street, Kazan, 420066, Russian \\ Federation
}

\begin{abstract}
The fourth-order ordinary differential spectral problem describing vertical eigenvibrations of a beam with two mechanical resonators attached to the ends is studied. This problem has positive simple eigenvalues and corresponding eigenfunctions. We define limit differential spectral problem and establish the convergence of the eigenvalues and eigenfunctions of the original spectral problem to the eigenvalues and eigenfunctions of the limit spectral problem as parameters of the attached resonators tending to infinity. The initial fourth-order ordinary differential spectral problem is approximated by the finite difference method. Theoretical error estimates for approximate eigenvalues and eigenfunctions are derived. Obtained theoretical results are illustrated by computations for model problem with constant coefficients. Theoretical and experimental results of this paper can be developed for the problems on eigenvibrations of complex mechanical constructions with systems of resonators.
\end{abstract}

\section{Introduction}

We investigate the vertical eigenvibrations of a beam of length $l$. Denote by $\rho(x), E(x)$, $S(x)$ and $J(x)$ the density, the elasticity modulus of the beam material, the square of transversal cut of the beam and the inertia moment of the cut with respect to its horizontal axis at the point $x \in \bar{\Omega}=[0, l], \Omega=(0, l)$. Assume that the ends $x=0$ and $x=l$ of the beam are elastically fixed by springs of stiffness $K$, also at points $x=0$ and $x=l$ of the beam loads of mass $M$ are joined. Then the vertical deflection $w(x, t)$ of the beam at a point $x$ at time $t$ satisfies the following system of partial differential equations

$$
\frac{\partial^{2}}{\partial x^{2}}\left(p \frac{\partial^{2} w}{\partial x^{2}}\right)+r \frac{\partial^{2} w}{\partial t^{2}}=0, x \in \Omega, t>0,
$$

\footnotetext{
* Corresponding author: sergey.solovev.kpfu@mail.ru
} 


$$
\begin{gathered}
\frac{\partial^{2} w(0, t)}{\partial x^{2}}=0, \frac{\partial^{2} w(l, t)}{\partial x^{2}}=0, t>0, \\
-\frac{\partial}{\partial x}\left(p(0) \frac{\partial^{2} w(0, t)}{\partial x^{2}}\right)-K w(0, t)=M \frac{\partial^{2} w(0, t)}{\partial t^{2}}, t>0, \\
\frac{\partial}{\partial x}\left(p(l) \frac{\partial^{2} w(l, t)}{\partial x^{2}}\right)-K w(l, t)=M \frac{\partial^{2} w(l, t)}{\partial t^{2}}, t>0
\end{gathered}
$$

with coefficients $p(x)=J(x) E(x), r(x)=S(x) \rho(x), x \in \bar{\Omega}, t>0$.

The eigenvibrations of the beam-resonators mechanical system are described by the deflection $w(x, t)=u(x) \sin (\omega t), x \in \bar{\Omega}, t>0$, with constant $\omega$. The system of partial differential equations (1)-(4) leads to the parameter fourth-order ordinary differential spectral problem: find $\lambda=\lambda(K, M)$ and $u(x)=u^{K, M}(x), x \in \bar{\Omega}$, satisfying the following equations

$$
\begin{gathered}
\left(p u^{\prime \prime}\right)^{\prime \prime}-\lambda r u=0, x \in \Omega, \\
u^{\prime \prime}(0)=u^{\prime \prime}(l)=\left(p(0) u^{\prime \prime}(0)\right)^{\prime}+(K-\lambda M) u(0)=\left(p(l) u^{\prime \prime}(l)\right)^{\prime}-(K-\lambda M) u(l)=0 .
\end{gathered}
$$

The parameter spectral problem (5), (6), has positive simple eigenvalues and corresponding orthonormal eigenfunctions. In this paper, we study limit properties as $K \rightarrow \infty$ with fixed $M$ and as $M \rightarrow \infty$ with fixed $K$ of eigenvalues and eigenfunctions of the parameter spectral problem (5), (6). The original spectral problem (5), (6), is approximated by the finite difference mesh scheme. The theoretical errors estimates for approximate eigenvalues and eigenfunctions for this mesh scheme are established.

Spectral approximations for compact operators are investigated in the papers [1-4]. Generalizations of spectral approximations for holomorphic Fredholm operator functions are derived in the papers $[5,6]$. Preconditioned iterative methods for solving linear spectral problems are proposed and investigated in the papers [7-14]. Iterative methods for solving spectral problems with nonlinear parameter are proposed and investigated in the papers [15-26]. Numerical algorithm without saturation for solving problems of mathematical physics and mechanics were constructed and investigated in [27-38]. This paper develops and generalizes results of the papers [1-6].

\section{Limit properties of the beam-resonators eigenvalue problem}

Introduce sufficiently smooth coefficients $p(x), r(x), x \in \bar{\Omega}$, and assume that there exist positive numbers $\alpha_{i}, \quad \beta_{i}, i=1,2$, satisfying the following conditions $\alpha_{1} \leq p(x) \leq \alpha_{2}, \quad \beta_{1} \leq r(x) \leq \beta_{2}, x \in \bar{\Omega}$. We also introduce nonnegative numbers $K$, $M$, and the following bilinear forms

$$
a(u, v)=\int_{0}^{l} p u^{\prime \prime} v^{\prime \prime} \mathrm{d} x, b(u, v)=\int_{0}^{l} r u v \mathrm{~d} x, c(u, v)=u(0) v(0)+u(l) v(l) .
$$


Problem (5), (6), has positive simple eigenvalues $\lambda_{m}=\lambda_{m}(K, M), m=1,2, \ldots$ and corresponding eigenfunctions $u_{m}=u_{m}^{K, M}, \quad m=1,2, \ldots$, satisfying the following conditions

$$
a\left(u_{m}, u_{n}\right)+K c\left(u_{m}, u_{n}\right)=\lambda_{m} \delta_{m n}, b\left(u_{m}, u_{n}\right)+M c\left(u_{m}, u_{n}\right)=\delta_{m n},
$$

for $m, n=1,2, \ldots$

We introduce the limit spectral problem: find $\mu$ and functions $v(x), x \in \bar{\Omega}$, satisfying the following equations

$$
\begin{gathered}
\left(p v^{\prime \prime}\right)^{\prime \prime}+\mu r v=0, x \in \Omega, \\
v(0)=v(l)=v^{\prime \prime}(0)=v^{\prime \prime}(l)=0 .
\end{gathered}
$$

The spectral problem (7), (8), has eigenvalues $\mu_{m}, m=1,2, \ldots$ and corresponding eigenfunctions $v_{m}, m=1,2, \ldots$

Theorem 1. The eigenvalues $\lambda_{m}(K, M), M \in[0, \infty), m=1,2, \ldots$, with fixed $K$, are continuous and decreasing. The eigenvalues $\lambda_{m}(K, M), K \in[0, \infty), m=1,2, \ldots$, with fixed $M$, are continuous and increasing. The properties of eigenfunctions are valid: $u_{m}^{K, M}(0) \neq 0, u_{m}^{K, M}(l) \neq 0, m=1,2, \ldots$

The results of this theorem follow from the papers [1-6].

If $w(x), x \in \bar{\Omega}$, is a continuous function, then we define the following norm

$$
\|w\|=\max _{x \in \bar{\Omega}}|w(x)| .
$$

Theorem 2. The properties are valid: 1$)$ if $K$ is fixed, then $\lambda_{m}(K, M) \rightarrow \mu_{m-2}$ as $M \rightarrow \infty, \quad \lambda_{1}(K, M) \rightarrow 0$ and $\lambda_{2}(K, M) \rightarrow 0$ as $M \rightarrow \infty,\left\|u_{m}^{K, M}-v_{m-2}\right\| \rightarrow 0$ as $M \rightarrow \infty ; 2)$ if $M$ is fixed, then $\lambda_{m}(K, M) \rightarrow \mu_{m}$ as $K \rightarrow \infty,\left\|u_{m}^{K, M}-v_{m}\right\| \rightarrow 0$ as $K \rightarrow \infty$.

The proof of this theorem develops results from the papers [1-6].

\section{Numerical experiments}

Define $\quad x_{j}=j h, j=0,1, \ldots, N, \quad h=l / N$. Set $p(-x)=p(x), \quad p(l+x)=p(l-x)$, $x \in \bar{\Omega}, \quad$ and $\quad$ denote $\quad p_{j}=p\left(x_{j}\right), \quad r_{j}=r\left(x_{j}\right), \quad y_{x, j}=\left(y_{j+1}-y_{j}\right) / h$, $y_{\bar{x}, j}=\left(y_{j}-y_{j-1}\right) / h$. We approximate spectral problem (5), (6), by the following mesh scheme of finite difference method

$$
\begin{aligned}
& \left(p y_{\bar{x} x}\right)_{\bar{x} x, j}-\lambda^{h} r_{j} y_{j}=0, j=2,3, \ldots, N-2, \\
& \frac{1}{h^{2}} p_{1} y_{\bar{x} x, 1}+\frac{1}{h} K y_{0}=\lambda^{h}\left(\frac{1}{2} r_{0} y_{0}+\frac{1}{h} M y_{0}\right),
\end{aligned}
$$




$$
\begin{gathered}
-\frac{1}{h}\left(p y_{\bar{x} x}\right)_{\bar{x}, 1}-\frac{1}{h^{2}} p_{1} y_{\bar{x} x, 1}=\lambda^{h} r_{1} y_{1}, \\
-\frac{1}{h}\left(p y_{\bar{x} x}\right)_{\bar{x}, N-1}-\frac{1}{h^{2}} p_{N-1} y_{\bar{x} x, N-1}=\lambda^{h} r_{N-1} y_{N-1}, \\
\frac{1}{h^{2}} p_{N-1} y_{\bar{x} x, N-1}+\frac{1}{h} K y_{N}=\lambda^{h}\left(\frac{1}{2} r_{N} y_{N}+\frac{1}{h} M y_{N}\right) .
\end{gathered}
$$

Let us denote $[y, z)=\sum_{i=0}^{N-1} h y_{i} z_{i},(y, z]=\sum_{i=1}^{N} h y_{i} z_{i}$.

Theorem 3. The finite difference spectral problem (9)-(13) has positive eigenvalues $\lambda_{1}^{h}<\lambda_{2}^{h}<\ldots<\lambda_{N+1}^{h}$, and corresponding orthonormal eigenvectors $y^{(m)}=\left(y_{0}^{(m)}, y_{1}^{(m)}, \ldots, y_{N}^{(m)}\right)^{\mathrm{T}}, m=1,2, \ldots, N+1$, satisfying the following relations

$$
\begin{gathered}
\frac{1}{2}\left[p y_{\bar{x} x}^{(m)}, y_{\bar{x} x}^{(n)}\right)+\frac{1}{2}\left(p y_{\bar{x} x}^{(m)}, y_{\bar{x} x}^{(n)}\right]+K y_{0}^{(m)} y_{0}^{(n)}+K y_{N}^{(m)} y_{N}^{(n)}=\lambda_{m}^{h} \delta_{m n}, \\
\frac{1}{2}\left[r y^{(m)}, y^{(n)}\right)+\frac{1}{2}\left(r y^{(m)}, y^{(n)}\right]+M y_{0}^{(m)} y_{0}^{(n)}+M y_{N}^{(m)} y_{N}^{(n)}=\delta_{m n},
\end{gathered}
$$

for $m, n=1,2, \ldots, N+1, \quad y_{-1}^{(m)}+y_{1}^{(m)}=0, \quad y_{N-1}^{(m)}+y_{N+1}^{(m)}=0$.

Theorem 4. The theoretical error estimates for approximate eigensolutions hold: $\left|\lambda_{m}^{h}-\lambda_{m}\right| \leq c h^{2}, \quad\left\|y^{(m)}-u^{(m)}\right\|_{h} \leq c h^{2}, \quad$ with $\quad$ a constant $\quad c \neq c(h)$, $\left\|u^{(m)}-y^{(m)}\right\|_{h}=\max _{j=0,1, \ldots, N}\left|u_{j}^{(m)}-y_{j}^{(m)}\right|$,

$\left[r y^{(m)}, u^{(m)}\right)+\left(r y^{(m)}, u^{(m)}\right]+2 M y_{0}^{(m)} u_{0}^{(m)}+2 M y_{N}^{(m)} u_{N}^{(m)}>0, \quad u_{j}^{(m)}=u_{m}\left(x_{j}\right)$, $j=0,1, \ldots, N, 1 \leq m<N+1$.

The theoretical results of Theorems 3 and 4 can be established with using results from [1-6]. 

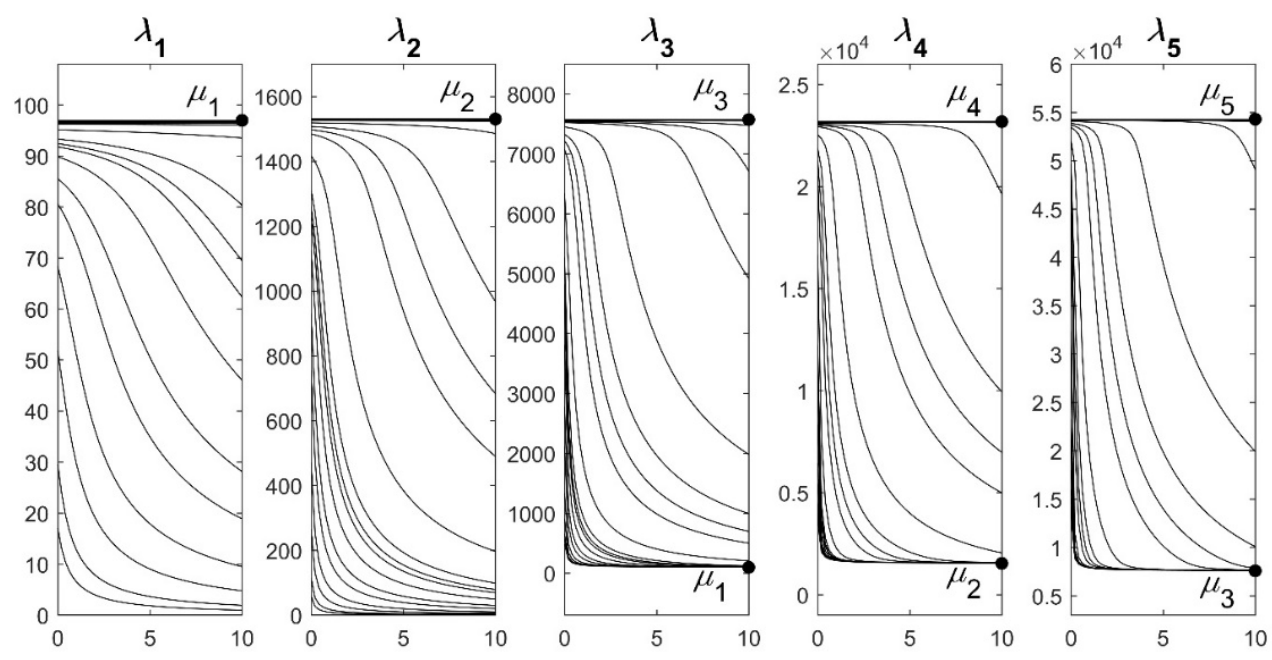

Fig. 1. Eigenvalues of the beam-resonators spectral problem.

Figure 1 shows the experimental results for a model problem. To illustrate Theorems 1 and 2, we solve the spectral problem (5), (6), for $M \in[0,2], K \in\left[0,10^{8}\right]$, and the following constant coefficients $p=1, r=1, x \in \bar{\Omega}, l=1$. We use the finite difference method (9)-(13) with $N=20$. Figure 1 shows the graphs of the eigenvalues $\lambda_{m}=\lambda_{m}(K, M), \quad M \in[0,10], \quad 1 \leq m \leq 5$, with fixed $K \in\left[0,10^{8}\right]$, the eigenvalues $\mu_{m}, 1 \leq m \leq 5$, of the spectral problem (7), (8). We can see that the obtained numerical results are consistent with Theorems 1 and 2: for fixed $K, \lambda_{m}(K, M) \rightarrow \mu_{m-2}$ as $M \rightarrow \infty, \quad m=1,2,3, \quad \lambda_{1}(K, M) \rightarrow 0$ and $\lambda_{2}(K, M) \rightarrow 0$ as $M \rightarrow \infty$, for fixed $M$, $\lambda_{m}(K, M) \rightarrow \mu_{m}$ as $K \rightarrow \infty, 1 \leq m \leq 5$. Theoretical and experimental results of this paper can be developed for the problems on eigenvibrations of complex mechanical constructions with systems of resonators.

This work was supported by Russian Foundation for Basic Research, project nos. 20-31-90086, 2008-01154.

\section{References}

1. Osborn J E 1975 Math. Comp. 29 (131) 712-25

2. Bramble J H and Osborn J E 1973 Math. Comp. 27 (123) 525-49

3. Knyazev A V and Osborn J E 2006 SIAM J. Numer. Anal. 43 (6) 2647-67

4. Sakurai T and Sugiura H 2003 J. Comput. Appl. Math. 159 119-28

5. Karma O 1996 Numer. Funct. Anal. Optim. 17 365-87

6. Karma O 1996 Numer. Funct. Anal. Optim. 17 389-408

7. Knyazev A V and Neymeyr K A 2003 Linear Algebra Appl. 358 (1-3) 95-114

8. Knyazev A V and Neymeyr K A 2003 Electr. Trans. Numer. Anal. 15 38-55

9. Neymeyr K A 2001 Linear Algebra Appl. 322 (1-3) 61-85

10. Neymeyr K A 2001 Linear Algebra Appl. 322 (1-3) 87-104 
11. Ovtchinnikov E E 2008 J. Comput. Phys. 227 (22) 9477-97

12. Ovtchinnikov E E 2008 SIAM J. Numer. Anal. 46 (5) 2567-92

13. Ovtchinnikov E E 2008 SIAM J. Numer. Anal. 46 (5) 2593-619

14. Ovtchinnikov E E 2006 SIAM J. Numer. Anal. 43 (6) 2668-89

15. Van Beeumen R, Meerbergen K and Michiels W 2013 SIAM J. Sci. Comput. 35 (1) A327-50

16. Beyn W-J, Effenberger C and Kressner D 2011 Numer. Math. 119 (3) 489516

17. Huang X, Bai Z and Su Y 2010 J. Comput. Appl Math. 28 (2) 218-34

18. Jarlebring E 2012 Linear Algebra Appl. 436 (10) 3943-53

19. Jarlebring E and Michiels W 2011 BIT 51 (4) 937-57

20. Sleijpen G L, Booten A G, Fokkema D R and van der Vorst H A 1996 BIT 36 (3) 595-633

21. Szyld D B and Xue F 2013 Numer. Math. 123 (2) 333-62

22. Andrew A L, Chu K E and Lancaster P 1995 Computing 55 91-111

23. Arbenz P and Gander W 1986 Computing 36 205-15

24. Asakura J, Sakurai T, Tadano H, Ikegami T and Kimura K 2009 JSIAM Letters $152-5$

25. Jarlebring E, Meerbergen K and Michiels W 2012 Numer. Math. 122 169-95

26. Su Y and Bai Z 2011 SIAM J. Matrix Anal. Appl. 32 201-16

27. Algazin S D and Babenko K I 1982 J. Appl. Math. Mech. 46 (6) 814-8

28. Algazin S D 1983 Siber. Math.J. 24 (2) 155-9

29. Algazin S D 1995 Comp. Math. Math. Phys. 35 (3) 321-30

30. Algazin S D 1995 Comp. Math. Math. Phys. 35 (4) 477-82

31. Algazin S D and Kiiko I A 2002 Dokl. Phys. 47 (3) 254-5

32. Algazin S D and Kiiko I A 2002 Dokl. Akad. Nauk. 383 (3) 343-6

33. Algazin S D 2008 Dokl. Math. 78 (2) 675-8

34. Algazin S D 2013 Math. Models Comp. Simulat. 5 (6) 520-6

35. Algazin S D 2013 J. Appl. Mech. Tech. Phys. 54 (2) 287-94

36. Algazin S D 2019 Dokl. Math. 99 (3) 260-2

37. Algazin S D 2006 J. Appl. Mech. Tech. Phys. 47 (3) 433-8

38. Algazin S D 2006 J. Appl. Mech. Tech. Phys. 47 (4) 573-81 